\title{
Precipitating hydrophobic injectable liquid embolization of intracranial vascular shunts: initial experience and technical note
}

\author{
Shriram Varadharajan, DM, ${ }^{1}$ Arvinda Hanumanthapura Ramalingaiah, DM, ${ }^{1}$ Jitender Saini, DM, ${ }^{1}$ \\ Arun Kumar Gupta, MD, ${ }^{1}$ B. Indira Devi, MCh, ${ }^{2}$ and Ullas V. Acharya, DM ${ }^{1}$
}

Departments of ${ }^{1}$ Neuroimaging and Interventional Radiology and ${ }^{2}$ Neurosurgery, National Institute of Mental Health and Neurosciences, Bangalore, Karnataka, India

\begin{abstract}
Precipitating hydrophobic injectable liquid (PHIL) is a newly available liquid embolic agent for endovascular therapy. It is nonadhesive and composed of a biocompatible polymer dissolved in dimethyl sulfoxide solvent and bonded covalently with iodine.

In this report, the authors present their preliminary experience using PHIL in the treatment of intracranial vascular shunts. Between July 2015 and December 2015, 11 patients underwent endovascular embolization using the PHIL embolic agent. Five patients had arteriovenous malformations, 4 had dural arteriovenous fistulas, 1 patient had a carotidcavernous fistula, and 1 patient had a pial arteriovenous fistula. Clinical features, angioarchitectural characteristics, procedural details, and periprocedural complications were included in the analysis.
\end{abstract}

Complete or near-complete obliteration of the nidus or fistulas was achieved in 8 of these patients. Partial embolization (approximately $80 \%$ in 2 and $30 \%$ in 1) was attained in the other 3 patients. Satisfactory venous penetration after nidal embolization was achieved in all patients. In 1 patient, the microcatheter could not be retrieved. No other major complications related to $\mathrm{PHIL}$ injection were noted during the procedure or periprocedural period. Clinical follow-up ranging from 8 months to 1 year showed good outcomes in all but 1 patient, who experienced an intraventricular hemorrhage 6 weeks after embolization.

PHIL appears to be an effective alternative embolic material with certain advantages over other available liquid embolic agents. Further studies with adequate follow-up are required to fully evaluate its safety and efficacy.

https://thejns.org/doi/abs/10.3171/2017.6.JNS16447

KEY WORDS precipitating hydrophobic injectable liquid; PHIL; embolization; vascular malformations; brain; surgical technique; interventional neurosurgery

$\mathrm{P}$ RECIPITATING hydrophobic injectable liquid (PHIL; MicroVention) is a newly available liquid embolic agent for endovascular use. PHIL contains a copolymer [poly(lactide co-glycolide) and poly(hydroxyethyl methacrylate)], which is suspended in dimethyl sulfoxide (DMSO). An iodine (triiodophenol) component is covalently bonded to the polymer, making it radiopaque. CE (Conformité Européenne) approval for its use in intracranial vascular malformations was obtained in July 2014. We describe our initial experience with the treatment of intracranial vascular shunts using PHIL.

\section{Methods}

Between July 2015 and December 2015, 11 patients underwent PHIL endovascular embolization. Five patients harbored arteriovenous malformations (AVMs), and 4 had dural arteriovenous fistulas (DAVFs). One had a carotidcavernous fistula $(\mathrm{CCF})$, and the remaining patient had a pial AVF.

\section{Clinical Presentation and Angioarchitecture}

Of the 5 AVMs, 3 were ruptured, resulting in intracra-

ABBREVIATIONS AVF = arteriovenous fistula; $\mathrm{AVM}=$ arteriovenous malformation; $\mathrm{CCF}=$ carotid-cavernous fistula; $\mathrm{DAVF}=$ dural $\mathrm{AVF} ; \mathrm{DMSO}=$ dimethyl sulfoxide; NBCA $=N$-butyl cyanoacrylate; $\mathrm{PHIL}=$ precipitating hydrophobic injectable liquid.

SUBMITTED February 21, 2016. ACCEPTED June 2, 2017.

INCLUDE WHEN CITING Published online December 1, 2017; DOI: 10.3171/2017.6.JNS16447. 
nial hemorrhage. The remaining 2 patients with AVM presented with seizures. The temporal and occipital lobes were the most common locations. Three AVMs were Spetzler-Martin Grade II. ${ }^{7}$ One patient had a diffuse AVM (Spetzler-Martin Grade V), for which embolization was performed as part of multiple staged procedures. Three AVMs were cortically located and exhibited superficial venous drainage. Prominent lenticulostriate feeders were seen in 1 patient, and a choroidal component with deep venous drainage was noted in another patient.

Among the 4 patients with DAVFs, 1 patient presented with an intracranial hemorrhage and the remaining 3 patients presented with headache, with additional orbital symptoms in 1 patient. Two DAVFs were located along the tentorium; 1 was in the right paracavernous region, and the final DAVF was located at the left transverse sigmoid junction. Two patients had Cognard Type IIa $+b,{ }^{2}$ AVFs, and direct cortical venous drainage was noted in the other 2 patients.

One patient presented with a recurrent posttraumatic left CCF after having undergone initial coil embolization. Another patient with a left parietal pial AVF presented with intracranial hemorrhage.

\section{Procedural Details}

Informed consent was obtained from all patients or their relatives prior to embolization. All embolization procedures were performed under general anesthesia with the patient intubated, and after embolization, the patient's blood pressure was maintained as per standard protocol to prevent perfusion pressure breakthrough.

DMSO-compatible microcatheters were used for embolization in all patients. After injecting DMSO to fill the dead space of the microcatheter, PHIL was injected using a push-pause technique. Percolation of the agent to the target areas was adequate despite a proximal position of the microcatheter in 2 cases. The mean duration of injection was 60 minutes (range 30-90 minutes), and the mean volume injected was $2.8 \mathrm{ml}$. The maximum injection duration was 90 minutes in a patient with a DAVF, in whom PHIL was injected through 2 separate feeders. In the patient with a pial AVF, coils were initially deployed to reduce the flow, and PHIL was subsequently injected. Another fistula in the same patient was embolized using $90 \% \mathrm{~N}$-butyl cyanoacrylate (NBCA) in view of high-flow fistulous communication. All procedures were carried out using 25\% PHIL.

\section{Complications}

No major complications related to the PHIL injection were noted. Adequate nidal penetration was achieved in all cases. Significant reflux $(>2 \mathrm{~cm})$ along the left middle meningeal artery occurred in 1 patient with a transverse sigmoid dural fistula, and the catheter became trapped. It was left in situ after repeated attempts to remove it failed. No other periprocedural complications were noted. The patients' postoperative stays were uneventful.

\section{Outcomes}

Angiographic outcome of complete or near-complete embolization was acheieved in 8 (73\%) patients (Fig. 1). Three patients with AVM had incomplete embolization (Fig. 2) due to persisting deep components (choroidal and lenticulostriate in 1 patient each, in whom $80 \%$ of the nidus was embolized). In 1 patient with a Spetzler-Martin Grade V AVM, approximately 30\% embolization was done as part of a staged procedure.

\section{Follow-Up}

All but 1 patient had good clinical outcomes (modified Rankin Scale score $\leq 2$ ) during follow-up (range 8 months to 1 year). A patient with a large AVM (Spetzler-Martin Grade V), who underwent staged treatment, presented with an intraventricular hemorrhage 6 weeks after PHIL
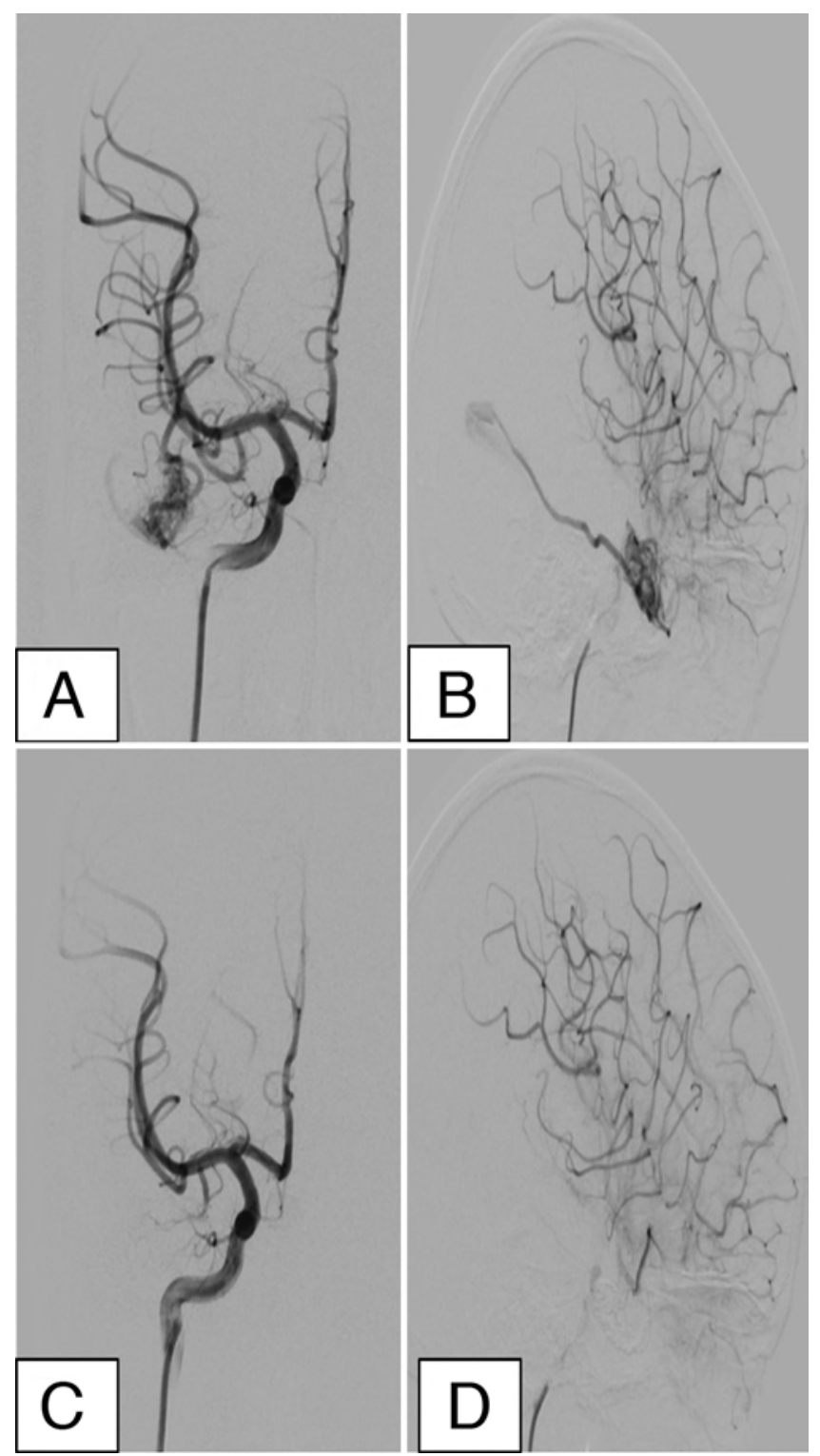

FIG. 1. Case 1. A and B: Preembolization anteroposterior (A) and lateral (B) angiograms obtained in a patient with a right temporal AVM (Spetzler-Martin Grade II) who underwent PHIL embolization. C and D: Postembolization anteroposterior (A) and lateral (B) angiograms showing complete obliteration of the nidus. 


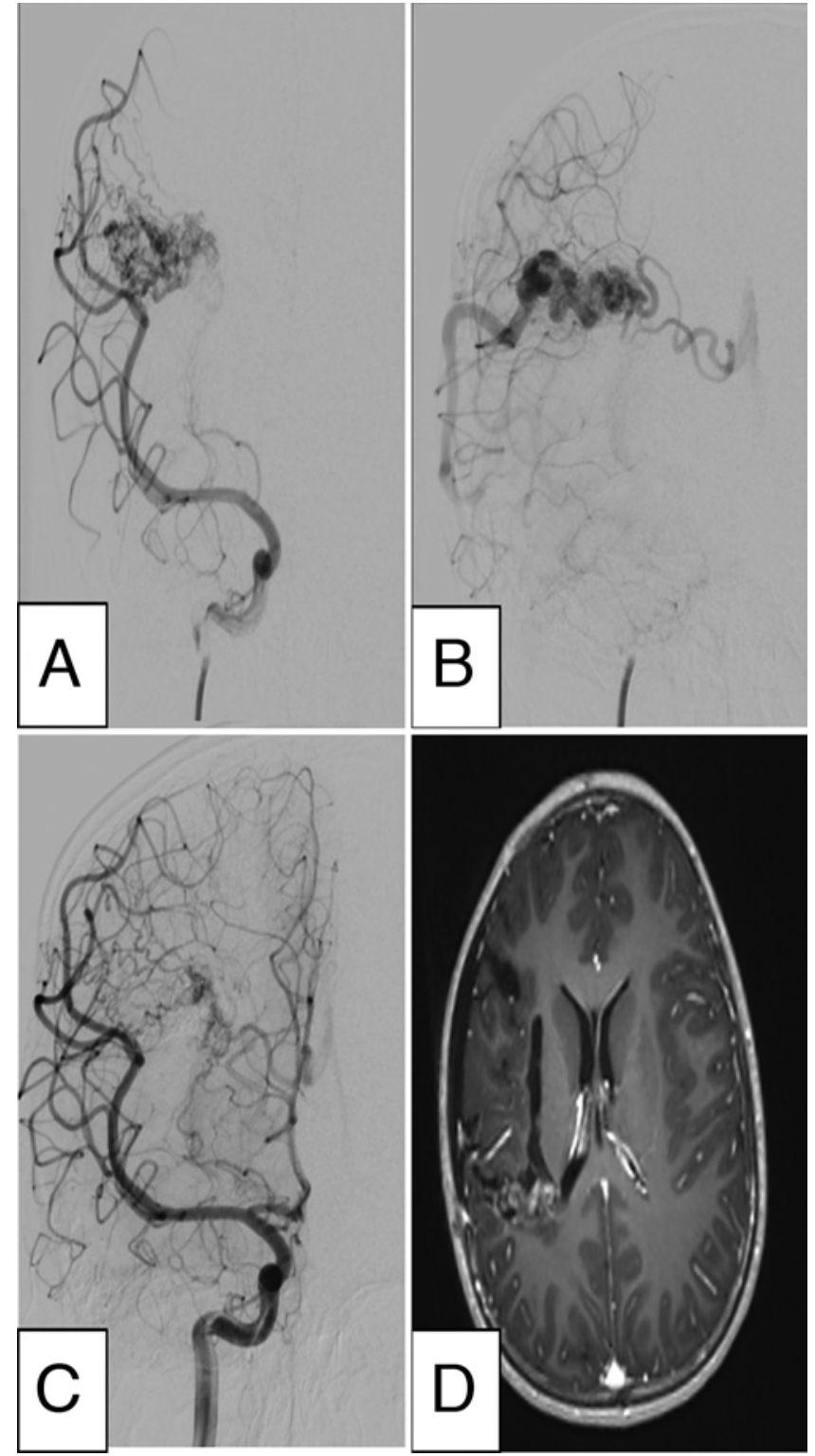

FIG. 2. Case 2. Images obtained in a patient with a right parietal AVM (Spetzler-Martin Grade III) who underwent PHIL embolization. A-C: Preembolization (A and B) and immediate postembolization (C) anteroposterior digital subtraction angiograms showing a persistent deep component of the nidus. D: Axial MR image obtained at the 3 -month follow-up, confirming a residual enhancing nidus.

embolization. This patient was managed conservatively. Imaging follow-up with either cross-sectional MR angiography or angiography after 6 months in the other patients with incomplete embolization showed a stable PHIL cast, with no obvious recanalization of the treated nidus. These patients were subsequently referred for stereotactic radiotherapy of the residual components. No recurrence or worsening of clinical symptoms was noted in the patients in whom complete embolization was achieved.

\section{Discussion}

We have described our initial experience with the use

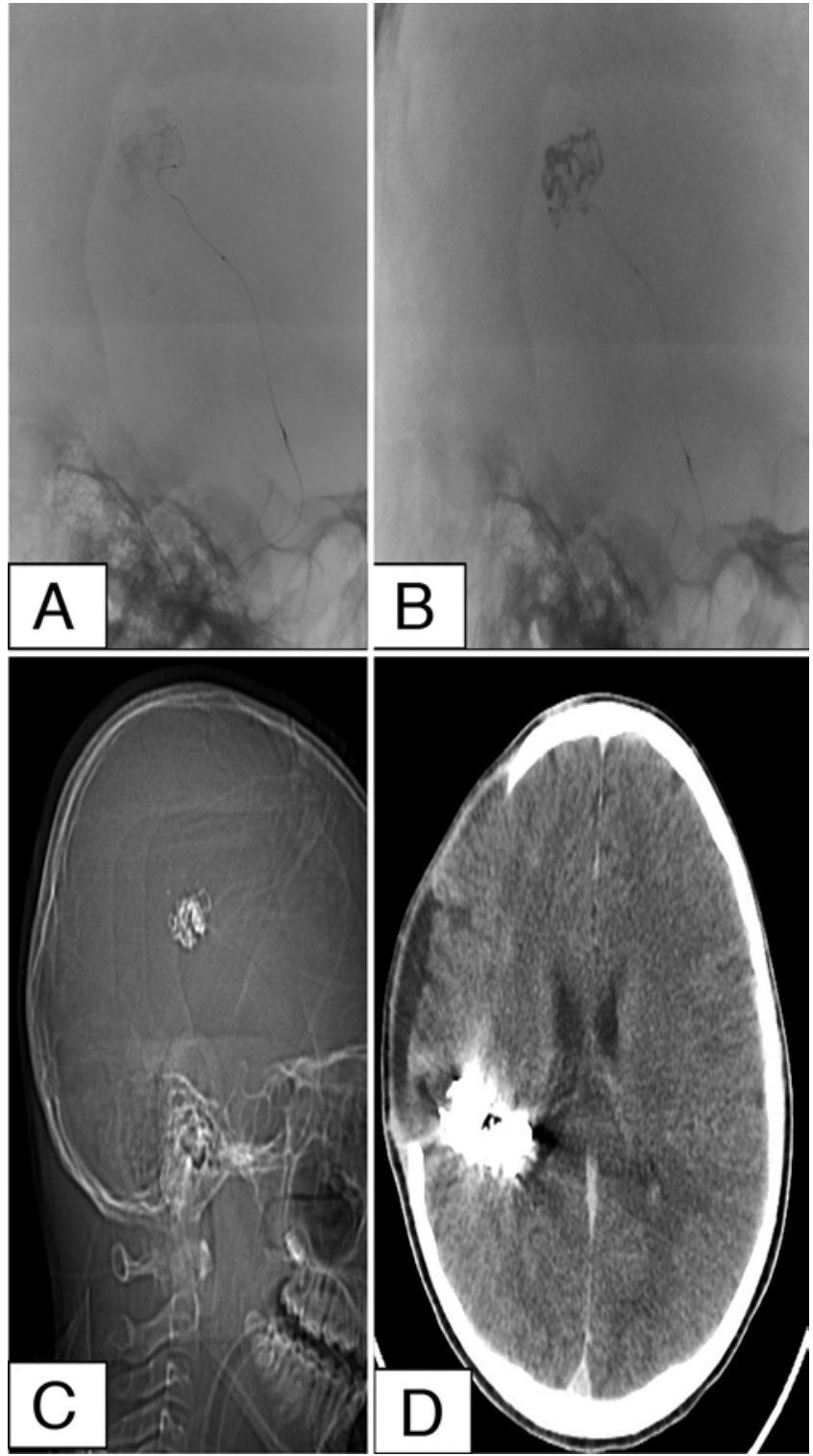

FIG. 3. Case 2. A and B: Lateral angiograms obtained in a patient with a right parietal AVM during the early $(A)$ and late $(B)$ phases of PHIL injection, showing the microcatheter tip throughout the procedure. $C$ and D: Scout (C) and axial (D) CT scans obtained after the procedure, showing the PHIL cast, with minimal streak artifacts.

of PHIL for the embolization of intracranial vascular malformations and shunts, relevant features of which are summarized in Table 1. Currently, Onyx (Medtronic) is the most widely used embolic agent for intracranial embolization. It is a nonadhesive copolymer that allows discontinuous, long injections of multiple feeders. ${ }^{1}$ Nidal penetration is also better with Onyx than earlier liquid embolic agents. ${ }^{5}$ PHIL is also a nonadhesive copolymer and thus retains these advantages of Onyx. Early studies on PHIL have shown favorable results in the embolization of intracranial dural fistulas ${ }^{4}$ and recently in brain AVMs. ${ }^{6}$ An iodine component (triiodophenol) is bound to a copolymer for radiopacity. PHIL advances into the vasculature as a block or column instead of layers and is less radiopaque, 
TABLE 1. Clinical presentation, angioarchitectural features, and outcomes of our patients using PHIL

\begin{tabular}{|c|c|c|c|c|c|c|}
\hline $\begin{array}{l}\text { Case } \\
\text { No. }\end{array}$ & Diagnosis & Location & Clinical Presentation & $\begin{array}{l}\text { Angioarchitectural } \\
\text { Grade* }\end{array}$ & $\begin{array}{l}\text { Amt of PHIL, ml } \\
\text { (time, mins) }\end{array}$ & Outcome \\
\hline 1 & AVM & Rt temporal & Ruptured & SM II & $0.9(60)$ & Complete embolization \\
\hline 2 & AVM & Rt parietal & Ruptured & SM III & $1.7(80)$ & Incomplete embolization (80\%) \\
\hline 3 & AVM & Rt frontoinsular & Ruptured & SM II & $5(70)$ & Incomplete embolization (80\%) \\
\hline 4 & AVM & Lt temporo-occipital & Unruptured (seizures) & SM V & $2.5(50)$ & Incomplete embolization (30\%) \\
\hline 5 & CCF & Lt-sided recurrence & Posttraumatic (orbital) & Barrow A† & $0.5(30)$ & Complete embolization \\
\hline 6 & DAVF & Lt transverse sigmoid & Ruptured & Cognard Ila+b & $2.6(70)$ & Complete embolization \\
\hline 7 & Pial AVF & Lt parietal & Ruptured & Double-hole fistula & $0.6(30)$ & Complete embolization \\
\hline 8 & DAVF & Tentorial & Unruptured (headache) & Cognard III & $6.5(70)$ & Complete embolization \\
\hline 9 & DAVF & $\begin{array}{l}\text { Rt paracavernous } \\
\text { area \& skull base }\end{array}$ & $\begin{array}{l}\text { Unruptured (headache } \\
\text { \& orbital symptoms) }\end{array}$ & Cognard IV & $5(90)$ & Complete embolization \\
\hline 10 & DAVF & Tentorial & Unruptured (headache) & Cognard lla+b & $4.4(70)$ & Complete embolization \\
\hline 11 & AVM & Lt occipital & Unruptured (seziures) & SM II & $1.2(40)$ & Complete embolization \\
\hline
\end{tabular}

Amt = amount; SM = Spetzler-Martin.

* At the time of presentation.

† Barrow DL, Spector RH, Braun IF, Landman JA, Tindall SC, Tindall GT: Classification and treatment of spontaneous carotid-cavernous sinus fistulas. J Neurosurg 62:248-256, 1985.

being visible only outside the microcatheter tip. However, from our experience, PHIL was optimally visible during its injection, and its cast could be clearly seen within the nidus. Furthermore, the microcatheter tip was clearly visible during the procedure, even within the PHIL cast. Formation of an initial plug was required, similar to Onyx, followed by antegrade flow into the nidus. Adequate venous penetration was obtained in all of our patients after embolization of the nidus. Layering of Onyx and the presence of tantalum within it (minimum size of tantalum is approximately $8-10 \mu \mathrm{m}$ ) precluded distal penetration of the Onyx into smaller vessels; however, studies have shown that PHIL can penetrate even into vessels smaller than $3 \mu \mathrm{m},{ }^{3}$ since iodine is covalently bonded to the copolymer.

PHIL is available in different concentrations $(25 \%$, $30 \%$, and $35 \%$ ) based on its viscosity; we administered the $25 \%$ concentration in all of our patients. High-flow fistulous communications were noted only in the patient with the pial AVF, and we used coils to reduce the flow before injecting PHIL. In the same patient, the other fistula was embolized using 90\% NBCA. There is a lack of literature regarding the use of PHIL in high-flow fistulas, and further studies are required. However, with this case, we were able to compare the postoperative PHIL cast with the NBCA cast on CT scans. Minimal streak artifacts were associated with the PHIL cast (Fig. 3). There are previous reports of cast migration during PHIL injection, ${ }^{4}$ which we did not encounter in our series. However, early venous filling was noted in 2 patients (Cases 6 and 10), and injection had to be stopped. After waiting a few minutes, we resumed the injection. Since PHIL travels as a column, there is a theoretical risk of early venous filling. However, it can be easily identified on the road map as a blob rather than plexiform, which would indicate the nidus. Thus, careful fluoroscopic monitoring during injection can prevent early inadvertent embolization of the venous side. On the other hand, this property allows PHIL to be injected in subop- timal positions in patients with tortuous anatomy where the fistulous point cannot be reached. In 2 of our patients, PHIL injection was performed in a suboptimal position, and we were still able to achieve satisfactory near-complete embolization of the fistula (Fig. 4).

Previous studies have not documented a retained microcatheter within the PHIL cast. In our series, the microcatheter could not be retrieved at the end of the procedure in a patient with a DAVF in whom embolization was performed through a middle meningeal artery feeder. However, we had allowed significant reflux $(>2 \mathrm{~cm})$ of the agent and had used a nondetachable microcatheter in that patient. The microcatheter was subsequently left in situ, and the patient was started on a regimen of antiplatelet medication after the procedure. No other major complications related to the PHIL injection were noted. We were able to achieve complete or near-complete embolization in $8(73 \%)$ of our patients. Incomplete embolizations in 3 patients were related to technical factors (deep feeders and staged embolization) unrelated to the PHIL injection. Clinical follow-up from a minimum of 8 months to 1 year revealed good outcomes in the majority of patients, with only 1 patient experiencing deterioration due to an intraventricular hemorrhage 6 weeks after embolization. Imaging follow-up over 6 months showed a stable PHIL cast in situ with no obvious recanalization of the treated portions (Fig. 5). None of the patients in whom near-complete or complete embolization was achieved exhibited any clinical recurrence of symptoms.

\section{Comparison of PHIL With Onyx}

PHIL and DMSO come in prefilled syringes that are relatively cumbersome to use compared with other syringes. There are occasions where PHIL has precipitated within the syringes over time, which is a major drawback. The agent contains covalently bonded iodine and lacks micronized tantalum compared with Onyx. Hence, it is 


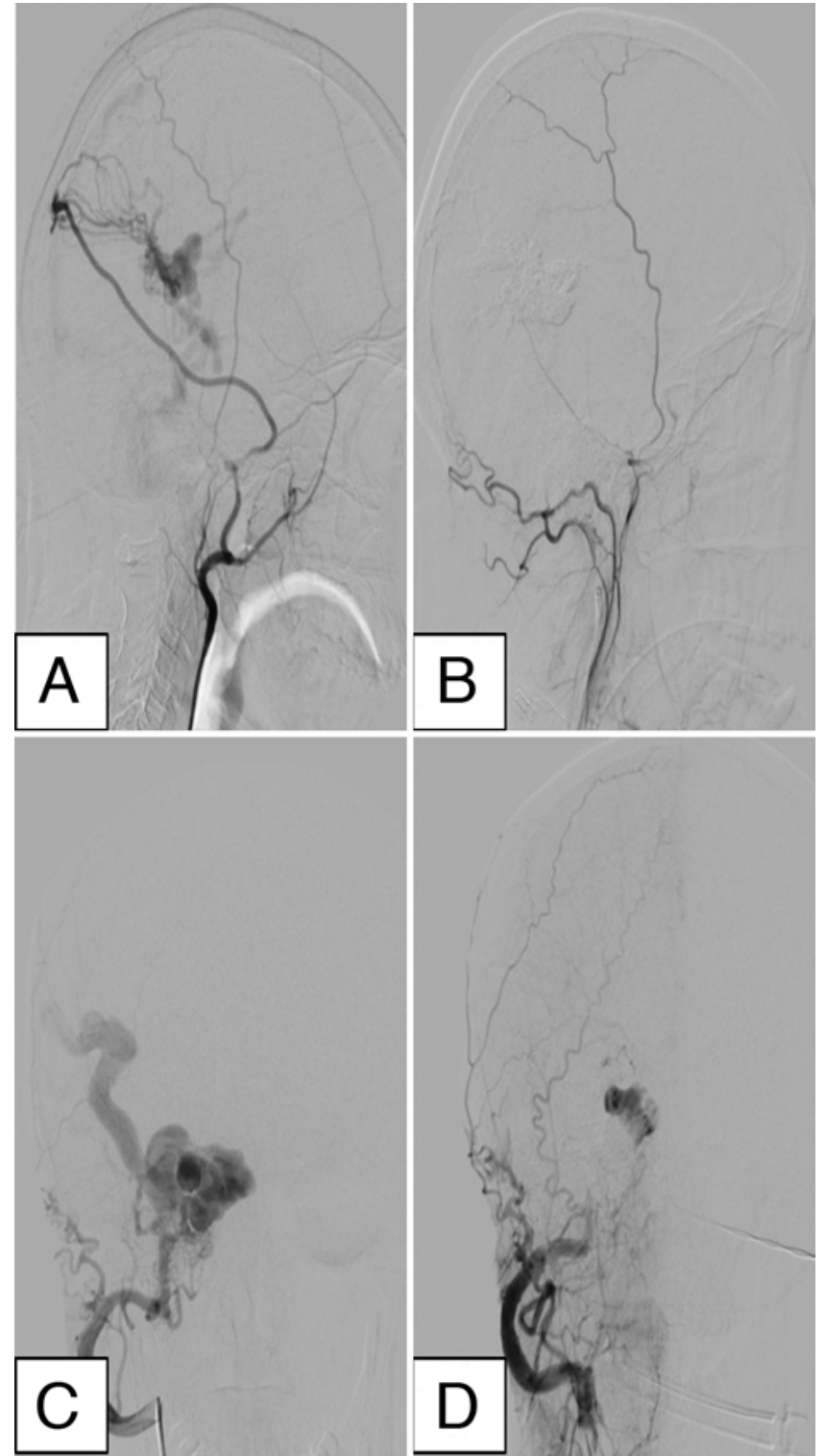

FIG. 4. Lateral angiograms obtained in a patient (Case 10) with a tentorial DAVF (A and $\mathbf{B}$ ) and anteroposterior angiograms in another patient (Case 9) with a right paracavernous DAVF (C and D) in whom PHIL embolization was carried out from a proximal suboptimal position. Preembolization ( $\mathrm{A}$ and $\mathrm{C}$ ) and postembolization ( $\mathrm{B}$ and $\mathrm{D}$ ) angiograms showing near-complete embolization of the fistulas.

more homogeneous and does not require prior shaking. PHIL is less radiopaque than Onyx and is often visualized only after it exits the microcatheter tip. Hence, caution must be exercised during the initial part of embolization to avoid inadvertent early venous embolization. However, this property is distinctly advantageous during the subsequent part of the procedure, when the microcatheter tip is clearly visible even amid a dense PHIL cast. PHIL travels as a column without any layering and achieves more distal penetration of the nidus due to its homogeneous composition. Oftentimes, a PHIL injection can be performed at a suboptimal proximal position, which is advantageous in cases of tortuous vessels precluding distal access. Shorter

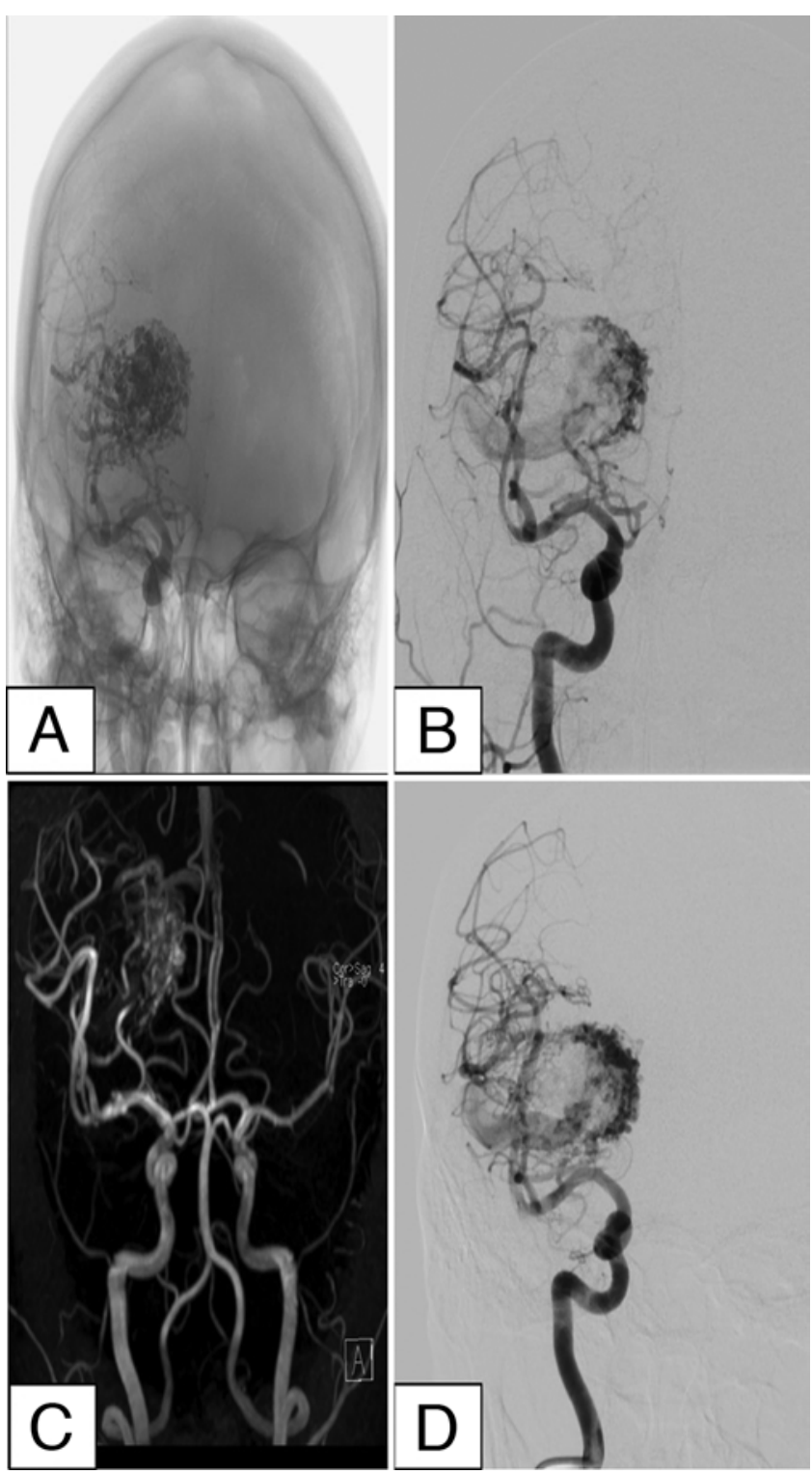

FIG. 5. Case 3. A and B: Anteroposterior angiograms obtained immediately after embolization (A) and at the 5-month follow-up (B) in a patient with a right frontal AVM (Spetzler-Martin Grade II), showing partial embolization. A persisting nidus is seen along the deep medial aspect. C and D: MR angiogram (C) and anteroposterior digital subtraction angiogram (D) obtained at the 1-year follow-up, showing a persisting residual nidus, with no obvious recanalization of the embolized portion.

intervals (30-60 seconds) are required during injection of PHIL than Onyx (2 minutes), which allow a greater margin for reflux $(2 \mathrm{~cm})$. The microcatheter could not be retrieved in only 1 patient in this series. Venous embolizations are often identified as distinct blobs and thus serve as a useful sign to stop the injection. Postembolization CT scans show fewer streak artifacts with PHIL than other embolic agents. Finally, since iodine is used as the radiopaque material PHIL and has a lower atomic number than tantalum, radiation factors can be altered for adequate visualization. This, combined with the reduced procedural time (reduced intervals between injections due to variability in 
percolation of PHIL), should reduce the effective radiation amount to the patient and operator. However, larger studies are required to validate this supposition.

\section{Conclusions}

We have described our initial experience with using PHIL in intracranial vascular shunts, and the immediate angiographic results appear to be favorable. The clinical follow-up of 8 months to 1 year is encouraging, with only 1 patient showing deterioration. PHIL casts appear stable, with no imaging evidence of recanalization over 6 months. Large, multicenter trials comparing the different embolic agents are needed to confirm the safety, efficacy, and durability of embolization.

\section{Acknowledgments}

We would like to thank Dr. Naci Kocer, İstanbul, Turkey, for proctoring our first patient who underwent PHIL embolization, and Mr. Vijay, from MicroVention, for coordinating the delivery of the PHIL material.

\section{References}

1. Choo DM, Shankar JJ: Onyx versus nBCA and coils in the treatment of intracranial dural arteriovenous fistulas. Interv Neuroradiol 22:212-216, 2016

2. Cognard C, Gobin YP, Pierot L, Bailly AL, Houdart E, Casasco A, et al: Cerebral dural arteriovenous fistulas: clinical and angiographic correlation with a revised classification of venous drainage. Radiology 194:671-680, 1995

3. Koçer N, Hanımoğlu H, Batur Ş, Kandemirli SG, Kızılkılıç $\mathrm{O}$, Sanus Z, et al: Preliminary experience with precipitating hydrophobic injectable liquid in brain arteriovenous malformations. Diagn Interv Radiol 22:184-189, 2016

4. Leyon JJ, Chavda S, Thomas A, Lamin S: Preliminary experience with the liquid embolic material agent PHIL (precipitating hydrophobic injectable liquid) in treating cranial and spinal dural arteriovenous fistulas: technical note. J Neurointerv Surg 8:596-602, 2016
5. Natarajan SK, Born D, Ghodke B, Britz GW, Sekhar LN: Histopathological changes in brain arteriovenous malformations after embolization using Onyx or N-butyl cyanoacrylate. Laboratory investigation. J Neurosurg 111:105-113, 2009

6. Samaniego EA, Kalousek V, Abdo G, Ortega-Gutierrez S: Preliminary experience with precipitating hydrophobic injectable liquid (PHIL) in treating cerebral AVMs. J Neurointerv Surg 8:1253-1255, 2016

7. Spetzler RF, Martin NA: A proposed grading system for arteriovenous malformations. J Neurosurg 65:476-483, 1986

\section{Disclosures}

The authors report no conflict of interest concerning the materials or methods used in this study or the findings specified in this paper.

\section{Author Contributions}

Conception and design: Varadharajan. Acquisition of data: Varadharajan. Analysis and interpretation of data: Varadharajan. Drafting the article: Varadharajan. Critically revising the article: Ramalingaiah, Saini. Reviewed submitted version of manuscript: Ramalingaiah, Saini. Approved the final version of the manuscript on behalf of all authors: Ramalingaiah. Administrative/ technical/material support: Saini, Gupta, Devi, Acharya. Study supervision: Ramalingaiah, Saini. Primary interventional neuroradiologist performing procedures: Ramalingaiah, Saini. Assisted in the procedures: Varadharajan, Acharya.

\section{Supplemental Information}

\section{Current Affiliations}

Dr. Varadharajan: CMC Hospital, Ludhiana, Punjab, India. Dr. Acharya: Manipal Hospital, Bangalore, India.

\section{Correspondence}

Arvinda Hanumanthapura Ramalingaiah, Department of Neuroimaging and Interventional Radiology, National Institute of Mental Health and Neurosciences, Bangalore, Karnataka 560029, India. email: aravind.radiology@gmail.com. 